\title{
Determinants of Intermittent Preventive Treatment of Malaria (IPTp-SP) Ideation among Women with at least one Child not older than 2 years in three Nigerian States
}

\author{
Article by Okoh, O.M ${ }^{1}$, Babalola, S. ${ }^{1,2}$, McCartney-Melstad, A. ${ }^{1}$, Tweedie, I. $^{1}$, Amosu, A. \\ $\mathrm{M}^{3}$ \\ ${ }^{1}$ Health Communication Capacity Collaborative Project and Johns Hopkins University \\ Center for Communications Program \\ ${ }^{2}$ Johns Hopkins University School of public health \\ ${ }^{3}$ Babcock University School of public and allied health \\ E-mail: mathewokoh@yahoo.com ${ }^{1}$
}

\begin{abstract}
Ideation is a set of ideas or ways of thinking that influence how people behave and how these ways of thinking are propagated within a community. Ideation has been successfully used to influence adoption of protective/preventive behavior in HIV and family planning interventions. Its use in the field of malaria is gradually gaining prominence but has been limited to bed net use.

This research investigated the demographic and behavioral factors that were associated with higher IPTp-SP ideation among women with a live birth in the two years preceding the survey. The study was quantitative in design and used cross sectional data that included 3205 women from three Nigerian states. Multilevel analysis was used to account for contextual factors.

Less than two-fifth of the women included in the analysis had higher IPTp-SP ideation. Factors that were found to be significantly associated with IPTp-SP ideation included uptake of IPTp-SP, use of ANC services, exposure to malaria messages, as well as the geographic location and prevalence of low educational status in the community where a woman lives.

Results from this study suggests that, (i) improved access to ANC services, could potentially increase IPTp-SP uptake through its effect on IPTp-SP ideation, (ii) dissemination of malaria messages especially those specific to IPTp-SP use remains a relevant option for improved utilization of IPTp-SP, (iii) there is need for interventions that specifically target communities with high prevalence of less than secondary education, in order to address those factors that negatively impact IPTp-SP ideation in such communities.
\end{abstract}

Keywords: Ideation, Malaria, Pregnancy, Nigeria, Prevention, Sulfadoxine-Pyrimethamine.

\section{Introduction}

Preventive health behavior is defined as any activity undertaken by an individual who believes $\mathrm{he} / \mathrm{she}$ is in good health in order to prevent disease(s) or detect disease in an asymptomatic stage (Kasl $\&$ Cobb, 1996). Several theories and models, including the ideational model of health behavior have been suggested to influence the adoption of protective behaviors or discontinuation of behaviors that have negative health impacts. Van de Kaa (1996) defined ideation as a set of views and ideas that people hold. Kincaid (2000b) expanded this definition when he described ideation as "new ways of thinking and the diffusion of those ways of thinking by means of social interactions in local, culturally homogeneous communities."

The ideational factors in Kincaid's proposed model of behavior change are categorized into three dimensions: cognitive, emotional, and social interactions. The cognitive dimension includes: attitudes, knowledge, perceived risk, subjective norms, and self-image. The emotional dimension includes preferences and self-efficacy. Finally, the social interaction dimension includes social influence, and personal advocacy (Kincaid, 2000a; Kincaid, 2000b). According to Kincaid (2000a), attitude is a function of the belief and values that people hold concerning a particular behavior. Subjective norms include what individuals think others expected of them and what they think other people are doing concerning the behavior. Self-image is the opinion or belief that people hold of themselves concerning 
a specific behavior. Finally, Kincaid described social interactions as those interpersonal processes by which individuals are influenced to behave in a particular way. These ideational factors interact synergistically in people to influence behavior change, such that the more relevant ideational elements an individual has, the higher the tendency for that individual to adopt the behavior in question (Kincaid, 2000a; Kincaid, 2000b). In 2015, a group of researchers modified the social component of Kincaid's original model to include interpersonal communication (e.g. spousal communication) and perceived social support. These two ideational factors were added to the model following the availability of evidence that support their association with the adoption of certain behavior (Babalola, John, Ajao, \& Speizer, (2015). The modified ideational model is shown in figure 1 below.

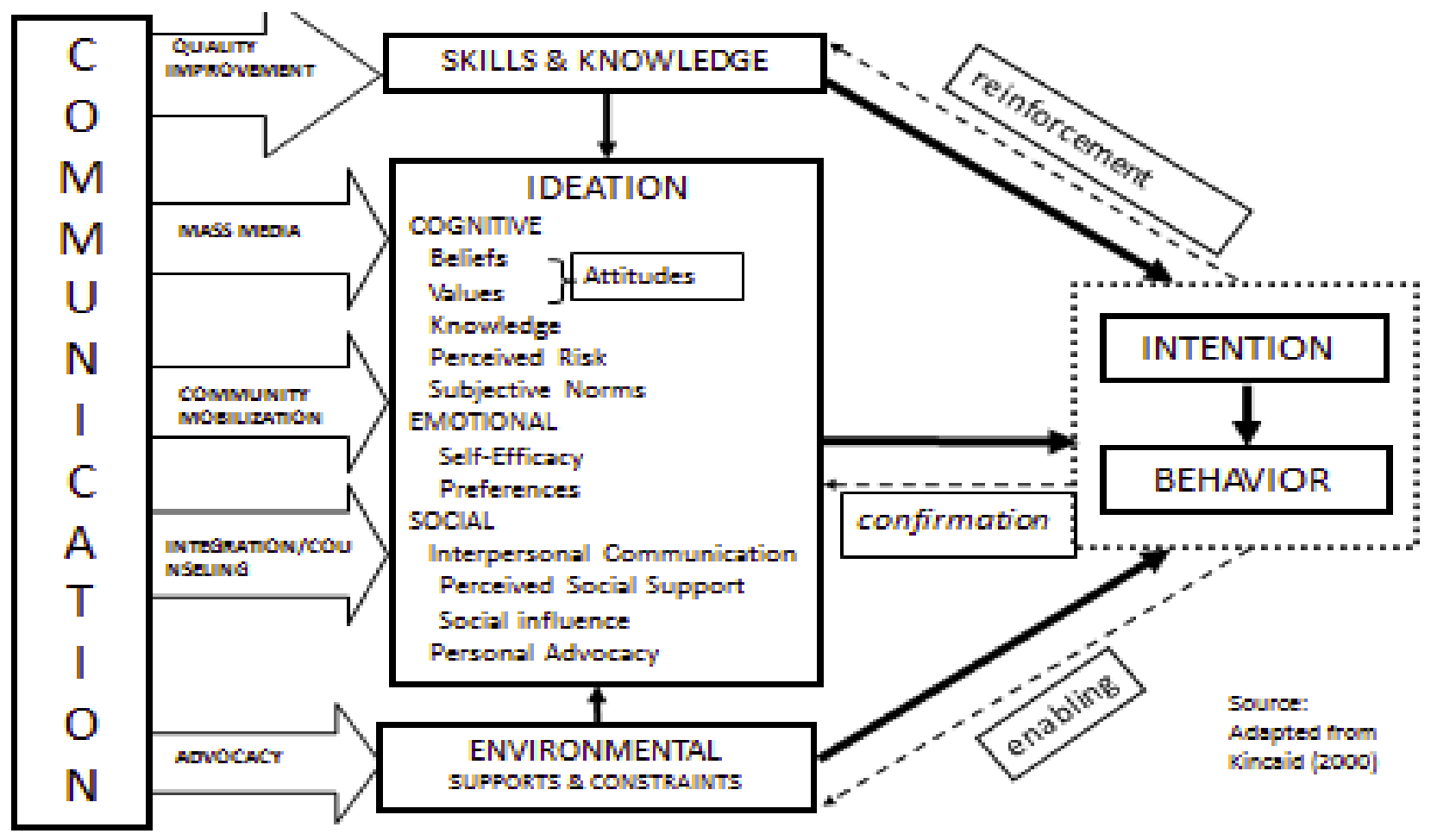

Figure 1. Ideation model of strategic communication and behavior change

The role of ideation in behavior change has been well documented particularly in the field of family planning. For example, spousal communication, perceived spousal approval of contraception, perceived normative support, perceived peer behaviors, perceived self-efficacy for contraceptive use, positive and negative attitudes about contraception have been reported to influence contraceptive use (Samandari, Speizer, \& O'Connell, 2010; Kaggwa, Diop, \& Storey, 2008; Ryan, Franzetta, \& Manlove, 2007; Bajwa, Bajwa, Ghai, Singh, \& Singh, 2012). Researchers have also demonstrated the synergistic effects of ideational factors in predicting behavior change. For example, Babalola, John, Ajao, \& Speizer, (2015) Babalola, Vondrasek, Brown, \& Traoré; (2001) Kincaid (2000a; 2000b) have linked increased contraceptive prevalence and intention to use contraception with increasing levels of ideation.

The use of ideation in predicting behavior change is relatively new in the field of malaria and available literature is restricted to net use and case management. Ricotta et al (2015) in an intervention study implemented in Tanzania reported that net ownership increased with increasing ideational level of participants. Similarly, Russell et al (2015) and Babalola et al (2016) have independently demonstrated an increasing likelihood of net use with increasing level of ideational factors among survey participants in Southeastern Nigeria and Liberia respectively. Furthermore, Do et al (2017) noted that ideational factors positively influenced fever treatment behavior among care givers of children under the age of 5 years in Madagascar, Mali and Nigeria. However, there is no literature describing the relationship between uptake of intermittent preventive treatment of malaria in pregnancy (IPTp) using sulphadoxine-pyrimethamine (SP) and ideation. 
Intermittent preventive treatment of malaria in pregnancy with sulphadoxine-pyrimethamine (IPTpSP) is an intervention designed to mitigate the effect of malaria infection in pregnant women. Pregnant women are especially vulnerable to the effects of malaria and are more likely than nonpregnant women to suffer severe consequences from malaria (Parise et al., 1998; WHO, 2004). Two facility-based studies conducted in South Eastern Nigeria indicated that prevalence of malaria was between $21 \%$ and $47 \%$ higher in pregnant women compared to their non-pregnant counterpart (Nduka, Egbu, Okafor, \&Nwaugo, 2006;Nnaji, Ikechebelu, \& Okafor, 2009). In Ghana, Tay and colleagues in 2013 estimated malaria prevalence to be 50\% higher in pregnant women compared to non-pregnant women. In most Sub-Saharan African countries including Nigeria where malaria is endemic, people often acquire immunity due to continued exposure to the malaria parasite. This often results in people becoming infected without showing symptoms (Sirima et al., 2006; Desai et al., 2007). A situation that could be dangerous for pregnant women living in a stable transmission environment as she may not be aware that she is infected until adverse consequences of the infection begin to manifest. Conversely, pregnant women in an environment of unstable transmission could quickly become severely sick with malaria if she gets infected due to lack of immunity (WHO, 2004).

Malaria infection during pregnancy is believed to contribute as much as $15 \%$ of maternal anaemia and 14\% of low birth weight (Steketee, Nahlen, Parise, \& Menendez, 2001; Steketee, Wirima, \&Campbell, 1996). According to Newman and colleagues, malaria in pregnancy is also responsible for $30 \%$ of preventable low birth weight, $70 \%$ of intrauterine growth retardation, $36 \%$ of premature deliveries, and $8 \%$ of infant mortality (Newman, Robalo, \& Quakyi, 2004). Malaria-related anaemia is estimated to cause as many as 10000 maternal deaths each year in Africa (Guyatt \& Snow, 2001). In Nigeria, malaria deaths account for $11 \%$ of maternal mortality (NMEP, NPoPC, NBS, \& ICF, 2016). In the light of these negative consequences of malaria in pregnant women, the World Health Organization (WHO) recommends that pregnant women in malaria endemic countries including Nigeria receive at least three doses of IPTp-SP (WHO, 2014) in order to safe guard the health of the pregnant woman and her unborn child from the effect of malaria infection. This IPTp-SP strategy has long been shown to be easily implementable, safe, affordable and efficacious in mitigating the effects of malaria infection among pregnant women (WHO, 2014)

In spite of the abundance of evidence regarding the usefulness of IPTp-SP in preventing poor pregnancy outcomes due to malaria infection during pregnancy, uptake of IPTp-SP among pregnant women in Nigeria still remains very low (NMEP, NPoPC, NBS, \& ICF, 2016; NPC \& ICF, 2014). This study therefore, intends to explore the IPTp-SP ideational profile of women who had a live birth in the two years preceding the survey. This study became necessary due to: (i) availability of literature linking behavior with ideation and (ii) the paucity of literature that specifically document IPTp-SP uptake and IPTp-SP ideation.

\section{Research objectives}

The objectives of this study were:

- To identify demographic factors associated with higher IPTp-SP ideation among women with a live birth in the last 2 years.

- To determine the relationship between IPTp-SP uptake and IPTp-SP ideation among women with a live birth in the last 2 years.

- To determine the influence of antenatal care (ANC) attendance on IPTp-SP ideation among women with a live birth in the last 2 years.

\section{Materials and methods}

\section{Design}

This analysis was based on a cross sectional household survey data, collected in 2015 as baseline for a project funded by the United States Agency for International Development (USAID). The project was designed to promote adoption of appropriate malaria behaviors and thereby decrease malaria disease burden in Nigeria through social and behavior change communication strategies. 


\section{Primary data collection plan}

Multistage cluster sampling design was used to select a representative sample of households with at least one child under the age of five years in three survey states (Akwa Ibom, Kebbi and Nasarawa). The sampling process involved selecting the local government area, the enumeration area (cluster), and then the household. Sample size for the study was based on proportion of the population with positive attitudes towards bed nets. Since such information was not available for any of the study states, it was assumed that this indicator was $50 \%$. This level of prevalence of positive attitudes is ideal for estimating the required sample size as it provides maximum variability. In addition, it was also assumed that program activities will result in an increase of 10 percentage points in the prevalence of positive attitudes towards bed nets. Based on these statistics and a power of 0.90 , a sample size of 1200 households per state was achieved after accounting for a $10 \%$ non-response rate

\section{Data collection}

Data was collected between July and October 2015. In each selected household, the mother of a child aged less than five years was invited to complete an interviewer-administered semi-structured questionnaire. One woman was randomly selected in households where there was more than one eligible woman. However, only women who had a live birth in the two years preceding the survey and currently in union were included in this analysis.

\section{Data analysis}

Multilevel modeling was used to assess the factors associated with higher IPTp-SP ideation among women who had a live birth in the two years preceding data collection. Multilevel analysis technique was chosen to account for the hierarchical nature of the data (individuals nested within clusters).

The main dependent (outcome) variable for this study was IPTp-SP related ideation among women who had a live birth in the two years preceding this survey. IPTp-SP ideation was constructed from knowledge of IPTp-SP, attitude towards ANC and IPTp, perceived IPTp-SP efficacy, perceived social norm about ANC, discussion of malaria and pregnancy with spouse, friends or relation and participation in decisions concerning her own health. Based on the number of Items used to construct IPTp-SP ideation, each woman had an IPTp-SP ideational score of between 0 and 6 . In order to categorize women as having lower or higher IPTp-SP ideation, we split the IPTp-Sp ideation construct at the median. Consequently, Women with IPTp-SP ideational score of less than 3 were considered as having lower IPTp-SP ideation, while those with a score of between 3 and 6 were categorized as having higher IPTp-SP ideation. The independent variables examined included: demographic variables, ANC attendance, IPTp-SP uptake during the most recent pregnancy, general malaria ideation, place of residence as well as the prevalence of poverty and low education level in the community of residence

\section{Multilevel models specification}

Three models with variables of interest were fitted using the melogit command in Stata 14. The first model (intercept only/empty model) was used to check if the data had justifiable evidence to assess random effect at the group level. The second model was used to examine the association between individual, household factors and IPTp-SP ideation, while the third model examined the effect of individual, household and community level factors on IPTp-SP ideation. All analysis were interpreted at $\mathrm{p}<0.05$.

\section{Ethical considerations}

Ethical approval for data collection was obtained from Johns Hopkins School of Public Health with IRB \#6234 as well as from the National Health Research Ethics Committee (NHREC) through the Federal Ministry of Health of Nigeria with approval number NHREC/01/01/2007-10/06/2015.

\section{Results}

The background characteristics of the 3205 women included in this study are presented in Table 1 . Four-fifths of the respondents were less than 35 years of age. One-third reported attaining at least 
secondary education, while close to half (44.9\%) reported no education. Respondents were almost evenly split between the Christian and Muslim faith. Most of the women included in the analysis were resident in a rural area.

Table 1. Percentage distribution of respondents' socio-demographic characteristics $(n=3205)$

\begin{tabular}{|l|l|}
\hline Socio-demographic Characteristics & $\mathbf{N}(\%)$ \\
\hline Age group & \\
$15-24$ & $974(30.4)$ \\
$25-34$ & $1609(50.2)$ \\
$35-44$ & $520(16.2)$ \\
$45+$ & $102(3.2)$ \\
\hline Religion & \\
Christian & $1575(49.1)$ \\
Muslim & $1553(48.5)$ \\
Other & $77(2.4)$ \\
\hline Parity & \\
$1-2$ & $1253(39.1)$ \\
$3-4$ & $1143(35.7)$ \\
$5+$ & $809(25.2)$ \\
\hline Education level & \\
None & $1439(44.9)$ \\
Primary & $787(24.6)$ \\
Secondary & $844(26.3)$ \\
Tertiary & $135(4.2)$ \\
\hline Type of place of residence & \\
Rural & $2470(77.1 \%)$ \\
Urban & $735(23.9 \%)$ \\
\hline Wealth index & \\
Poorest & $669(20.9)$ \\
Second & $642(20.0)$ \\
Middle & $653(20.4)$ \\
Fourth & $626(19.5)$ \\
Richest & $615(19.2)$ \\
\hline State of residence & $961(30.0)$ \\
Akwa Ibom & $1070(33.4)$ \\
Kebbi & $1174(36.6)$ \\
Nasarawa & \\
\hline
\end{tabular}

\section{Bivariate analysis of IPTp ideation and respondents' characteristics}

About four in ten $(39.8 \%)$ women had higher IPTp-SP ideation in this population. With the exception of age and parity, there was significant variation in the prevalence of IPTp-SP ideation by other background characteristics examined in this study (Table 2). Women of the Christian faith were significantly more likely to report higher IPTp-SP ideation compared to women of other faith. Urban women were also more likely to have higher IPTp-SP ideation compared to their rural counterpart. Education and wealth status exhibited a dose-response relationship with IPTp-SP ideation, such that as educational status or wealth status increased, the proportion of women who reported higher IPTpSP ideation also increased. Similarly, high IPTp ideation varied significantly by ANC attendance status and uptake of IPTp-SP status. The prevalence of high ideation was highest among women who reported a minimum of four ANC visit compared to those who did not attend ANC at all or attended ANC less than four times. The proportion of higher IPTp-SP ideation was significantly higher among women who reported uptake of a minimum of three doses of IPTp-SP compared to those who received less than three doses. 
DOI: $10.21522 /$ TIJPH.2013.06.01.Art010

ISSN: $2520-3134$

Table 2. Bivariate analysis of IPTp Ideation and respondents characteristics $(n=3205)$

\begin{tabular}{|c|c|c|c|}
\hline Characteristics & $\begin{array}{l}\text { Proportion with } \\
\text { lower ideation }\end{array}$ & $\begin{array}{l}\text { Proportion with } \\
\text { higher ideation }\end{array}$ & $\begin{array}{l}\text { Differences } \\
\text { with groups } \\
\text { (p value) }\end{array}$ \\
\hline $\begin{array}{l}\text { Age group } \\
15-24 \\
25-34 \\
35-44 \\
45\end{array}$ & $\begin{array}{l}63.55 \\
58.73 \\
58.08 \\
60.78 \\
\end{array}$ & $\begin{array}{l}36.45 \\
41.27 \\
41.92 \\
39.22 \\
\end{array}$ & 0.072 \\
\hline $\begin{array}{l}\text { Religion } \\
\text { Christian } \\
\text { Muslim } \\
\text { Other }\end{array}$ & $\begin{array}{c}49.02 \\
70.64 \\
76.62 \\
\end{array}$ & $\begin{array}{r}50.98 \\
29.36 \\
23.38 \\
\end{array}$ & $<0.001$ \\
\hline $\begin{array}{l}\text { Parity } \\
1-2 \\
3-4 \\
5+ \\
\end{array}$ & $\begin{array}{l}60.10 \\
59.58 \\
61.06 \\
\end{array}$ & $\begin{array}{l}39.90 \\
40.42 \\
38.94 \\
\end{array}$ & 0.803 \\
\hline $\begin{array}{l}\text { Education level } \\
\text { None } \\
\text { Primary } \\
\text { Secondary } \\
\text { Tertiary } \\
\end{array}$ & $\begin{array}{l}71.49 \\
56.85 \\
49.51 \\
46.94 \\
\end{array}$ & $\begin{array}{l}28.70 \\
44.22 \\
52.13 \\
56.30 \\
\end{array}$ & $<0.001$ \\
\hline $\begin{array}{l}\text { Type of place of } \\
\text { residence } \\
\text { Rural } \\
\text { Urban }\end{array}$ & $\begin{array}{l}61.21 \\
56.60\end{array}$ & $\begin{array}{l}38.79 \\
43.40 \\
\end{array}$ & 0.025 \\
\hline $\begin{array}{l}\text { Wealth index } \\
\text { Poorest } \\
\text { Second } \\
\text { Middle } \\
\text { Fourth } \\
\text { Richest }\end{array}$ & $\begin{array}{l}77.43 \\
64.64 \\
58.81 \\
52.40 \\
46.02 \\
\end{array}$ & $\begin{array}{l}22.57 \\
35.36 \\
41.19 \\
47.60 \\
53.90 \\
\end{array}$ & $<0.001$ \\
\hline $\begin{array}{l}\text { State of residence } \\
\text { Akwa Ibom } \\
\text { Kebbi } \\
\text { Nasarawa } \\
\end{array}$ & $\begin{array}{l}47.03 \\
78.97 \\
53.75 \\
\end{array}$ & $\begin{array}{l}52.97 \\
21.03 \\
46.25 \\
\end{array}$ & $<0.001$ \\
\hline $\begin{array}{l}\text { Community poverty rate } \\
\text { Low } \\
\text { Medium } \\
\text { High }\end{array}$ & $\begin{array}{l}47.06 \\
59.48 \\
73.59 \\
\end{array}$ & $\begin{array}{l}52.94 \\
40.52 \\
26.41 \\
\end{array}$ & $<0.001$ \\
\hline $\begin{array}{l}\text { Community literacy rate } \\
\text { Low } \\
\text { Medium } \\
\text { High }\end{array}$ & $\begin{array}{l}45.58 \\
57.33 \\
76.72 \\
\end{array}$ & $\begin{array}{l}54.42 \\
42.67 \\
23.28 \\
\end{array}$ & $<0.001$ \\
\hline $\begin{array}{l}\text { ANC attendance } \\
\text { Did not attend any ANC } \\
\text { Attended ANC less than } \\
4 \text { times } \\
\text { Attended ANC at least } 4 \\
\text { times }\end{array}$ & $\begin{array}{l}77.83 \\
64.55 \\
47.24\end{array}$ & $\begin{array}{l}22.17 \\
35.45 \\
52.76\end{array}$ & $<0.001$ \\
\hline
\end{tabular}




\begin{tabular}{|l|l|l|l|}
\hline Uptake of IPTp-SP & & & $<0.001$ \\
Did not take IPTp-SP & & & \\
Took less than 3 doses & & 34.29 & \\
of IPTp-SP & 66.42 & 51.16 & \\
Took at least 3 doses of & 49.88 & 53.10 & \\
IPTp-SP & 47.23 & & \\
\hline Malaria ideation & & 29.31 & \\
Low & 70.69 & 43.37 & \\
Medium & 56.63 & 37.29 & \\
High & 62.71 & & \\
\hline
\end{tabular}

\section{Individual and contextual factors associated with higher IPTp-SP ideation}

Table 3 presents the result of multilevel logistic regression modeling of factors that were independently associated with higher IPTp-SP ideation. The empty model (model 1) indicated significant variability in the odds of higher IPTp-SP ideation between communities $\left(\sigma^{2}=.52, p<0.001\right)$. The intra-cluster correlation also indicated that $13.6 \%$ of the variation in the outcome variable was due to unmeasured community factors. In model 2 we adjusted for individual level factors to examine their association with higher IPTp-SP ideation. Results from this model indicated a significant relationship between household wealth index, ANC attendance, IPTp-SP uptake, exposure to malaria messages, religion and higher IPTp-SP ideation. The variability of the outcome variable across communities was reduced in this model, although it was still significant. Unmeasured community level factors accounted for just $3 \%$ of the variation in the outcome variable as indicated by the intracluster correlation.

In model 3, we adjusted for individual and community variables. In this model, ANC attendance, IPTp-SP uptake, state of residence, exposure to malaria messages and prevalence of women with less than a secondary education in a community were found to be significantly associated with higher IPTp-SP ideation. There was a positive dose-response relationship between IPTp-uptake, ANC attendance and higher IPTp-SP ideation. Such that women who reported receiving at least three doses of IPTp-SP and those who received one or two doses were 63\% ( $\mathrm{p}<0.001)$ and $32 \%(\mathrm{p}=0.023)$ more likely to have higher IPTp-SP ideation compared to women who did not receive any dose of IPTp-SP. Similarly, women who reported attending ANC clinic at least four times were more than twice as likely to have higher IPTp-SP ideation compared to those who did not attend ANC. Unlike IPTp-SP uptake and ANC attendance, there was an inverse relationship between higher IPTp-SP ideation and the prevalence of women with less than a secondary education in a cluster. The higher the proportion of women with less than a secondary education in a cluster, the less likely it is for women residing in such cluster to have higher IPTp-SP ideation. Women who reported to have been exposed to malaria prevention messages in the 12 months preceding the survey were $22 \%(\mathrm{p}=0.03)$ more likely to have higher IPTp-SP ideation compared to those who reported not being exposed to malaria prevention messages.

State of residence also exhibited significant relationship with the outcome variable. Women resident in Kebbi State were 39\% less likely to have higher IPTp-SP ideation compared to women from Akwa Ibom State. Respondents from Nasarawa were however, not significantly different from their counterpart resident in Akwa Ibom State.

Educational status, religion, household wealth index and rural-urban residence did not appear to have a significant relationship with IPTp-SP ideation among this population. The intra-cluster correlation from this model after adjusting for individual and community level variables was further reduced to $1.5 \%$. 
DOI: $10.21522 /$ TIJPH.2013.06.01.Art010

ISSN: $2520-3134$

Table 3. Multilevel analysis of factors independently associated with IPTp-SP ideation

\begin{tabular}{|c|c|c|c|}
\hline Respondents characteristics & $\begin{array}{l}\text { Model } 1 \\
\text { intercept only } \\
\text { model }\end{array}$ & $\begin{array}{l}\text { Model } 2 \\
\text { individual } \\
\text { variables }\end{array}$ & $\begin{array}{l}\text { Model } 3 \\
\text { Community \& } \\
\text { individual variables }\end{array}$ \\
\hline Fixed effects & & & OR $(95 \% \mathrm{CI})$ \\
\hline $\begin{array}{l}\text { Age group } \\
15-24 \text { (ref) } \\
25-34 \\
35-44 \\
45+\end{array}$ & & $\begin{array}{l}1.00 \\
.92(.76-1.12) \\
.97(.75-1.26) \\
.96(.60-1.53) \\
\end{array}$ & $\begin{array}{l}1.00 \\
.91(.75-1.10) \\
.95(.74-1.24) \\
.96(.60-1.52) \\
\end{array}$ \\
\hline $\begin{array}{l}\text { Parity } \\
1-2 \text { (ref) } \\
3-4 \\
5+\end{array}$ & & $\begin{array}{l}1.00 \\
1.00(.83-1.20) \\
1.13(.90-1.41)\end{array}$ & $\begin{array}{l}1.00 \\
.99(.82-1.19) \\
1.12(.90-1.41)\end{array}$ \\
\hline $\begin{array}{l}\text { Religion } \\
\text { Christian (ref) } \\
\text { Muslim } \\
\text { Other }\end{array}$ & & $\begin{array}{l}1.00 \\
.58(.47-.71)^{* * * *} \\
.77(.41-1.44)\end{array}$ & $\begin{array}{l}1.00 \\
.79(.61-1.02) \\
1.16(.62-2.20) \\
\end{array}$ \\
\hline $\begin{array}{l}\text { Education level } \\
\text { None (ref) } \\
\text { Primary } \\
\text { Secondary } \\
\text { Tertiary } \\
\end{array}$ & & $\begin{array}{l}1.00 \\
.96(.76-1.20) \\
1.13(.89-1.45) \\
1.19(.77-1.84) \\
\end{array}$ & $\begin{array}{l}1.00 \\
.83(.66-1.05) \\
1.02(.80-1.31) \\
1.04(.67-1.62) \\
\end{array}$ \\
\hline $\begin{array}{l}\text { Wealth index } \\
\text { Poorest (ref) } \\
\text { Second } \\
\text { Middle } \\
\text { Fourth } \\
\text { Richest }\end{array}$ & & $\begin{array}{l}1.00 \\
1.32(.100-1.74)^{*} \\
1.40(1.06-1.86)^{*} \\
1.53(1.14-2.06)^{* *} \\
1.66(1.21-2.28)^{* *}\end{array}$ & $\begin{array}{l}1.00 \\
1.19(.90-1.57) \\
1.16(.86-1.56) \\
1.20(.86-1.66) \\
1.25(.87-1.79) \\
\end{array}$ \\
\hline $\begin{array}{l}\text { ANC attendance } \\
\text { Did not attend ANC } \\
\text { Attended ANC less than } 4 \\
\text { times } \\
\text { Attended ANC at least } 4 \\
\text { times }\end{array}$ & & $\begin{array}{l}1.00 \\
1.24(.96-1.59) \\
2.24(1.81-2.77)^{* * *}\end{array}$ & $\begin{array}{l}1.00 \\
1.16(.90-1.50) \\
2.08(1.68-2.57)^{* * *}\end{array}$ \\
\hline $\begin{array}{l}\text { IPTp-SP uptake } \\
\text { Did not receive IPTp-SP } \\
\text { Received less than } 3 \text { doses of } \\
\text { IPTp-SP } \\
\text { Received at least } 3 \text { doses of } \\
\text { IPTp-SP }\end{array}$ & & $\begin{array}{l}1.00 \\
1.43(1.13-1.83)^{* *} \\
1.62(1.32-1.99)^{* * *}\end{array}$ & $\begin{array}{l}1.00 \\
1.32(1.04-1.69)^{*} \\
1.63(1.32-2.00)^{* * *}\end{array}$ \\
\hline $\begin{array}{l}\text { Malaria ideation } \\
\text { Low (ref) } \\
\text { Medium } \\
\text { High } \\
\end{array}$ & & $\begin{array}{l}1.00 \\
1.23(.91-1.65) \\
1.19(.88-1.62) \\
\end{array}$ & $\begin{array}{l}1.00 \\
1.23(.91-1.65) \\
1.31(.96-1.78) \\
\end{array}$ \\
\hline $\begin{array}{l}\text { Exposed to malaria } \\
\text { prevention messages } \\
\text { No (ref) } \\
\text { Yes }\end{array}$ & & $\begin{array}{l}1.00 \\
1.26(1.06-1.50)^{* *}\end{array}$ & $\begin{array}{l}1.00 \\
1.22(1.02-1.45)^{*}\end{array}$ \\
\hline
\end{tabular}




\begin{tabular}{|l|l|l|l|}
\hline & & & \\
\hline $\begin{array}{l}\text { Type of place of residence } \\
\text { Urban (ref) }\end{array}$ & & & 1.00 \\
Rural & & & $1.09(.87-1.36)$ \\
\hline $\begin{array}{l}\text { State of residence } \\
\text { Akwa Ibom (ref) } \\
\text { Kebbi }\end{array}$ Nasarawa & & 1.00 \\
\hline $\begin{array}{l}\text { Prevalence of poor } \\
\text { households in Community } \\
\text { wealth status }\end{array}$ & & & $.61(.40-.94)^{*}$ \\
Low (ref) & & & $1.14(.85-1.53)$ \\
Medium & & & \\
High & & & 1.00 \\
\hline Prevalence of low education & & $.82(.64-1.03)$ \\
in Community & & $.94(.66-1.32)$ \\
\hline Low (ref) & & & \\
Medium & & & 1.00 \\
High & & & $.76(.60-.96)^{*}$ \\
\hline & & & $.61(.43-.86)^{* *}$ \\
\hline Random effects & & & \\
\hline Community variance (SE) & $0.52(0.09)^{* * *}$ & $0.11(.04)^{* * *}$ & $0.05(0.03)^{*}$ \\
\hline ICC (\%) & 13.6 & 3.0 & 1.5 \\
\hline & & & \\
\hline Model fit statistics & & -1928.80 \\
\hline Log-likelihood & & & 3917.61 \\
\hline AIC & & & \\
\hline & & & \\
\hline & & & \\
\hline
\end{tabular}

$* * * \mathrm{p}<0.001, * * \mathrm{p}<0.01, * \mathrm{p}<0.05$

\section{Discussion}

This study examined the factors associated with higher IPTp-SP ideation among women in union who had a live birth in the two years preceding the survey. Multilevel technique was used to explore the roles of individual demographic, behavioral and neighborhood factors in IPTp-SP ideation. There is an abundance of literature on the relationship between ideation and behavior, but this is the first study to examine the role of behavior (ANC attendance and IPTp-SP uptake) in ideation.

The prevalence of higher IPTp-SP ideation was low in this population but was found to be significantly associated with some characteristics of the respondents. Findings from this present study indicated a dose-response association between uptake of IPTp-SP and higher IPTp-SP ideation. Specifically, the more the number of IPTp-SP doses a woman received, the higher her IPTp-SP ideation. While earlier research had suggested that behaviors can over time influence ideation (Sutton, 2004), this is the first study to examine the effect of a specific behavior with its corresponding ideation. On the flip side however, several researchers have shown how ideation influence the performance of specific behaviors. For example, Ricotta et al (2015) in a study implemented in Tanzania reported that net ownership increased with increasing ideational level of participants. Similarly, Russell et al (2015) and Babalola et al (2016) have demonstrated an increasing likelihood of net use with increasing level of ideational factors among survey participants in Southeastern Nigeria and Liberia respectively. Furthermore, Kincaid (2000a; 2000b) Babalola, Vondrasek, Brown, 
\& Traoré; (2001) independently demonstrated that increased contraceptive prevalence and intention to use contraception were associated with increasing levels of ideation.

Women who had a minimum of four ANC visits were more than twice as likely to have higher IPTp-SP ideation compared to those who did not attend ANC. This finding might not be unconnected with the relationship between ANC attendance and IPTp-SP uptake. ANC is the mechanism recommended by the WHO for implementation of the IPTp-SP strategy, to ensure that pregnant women have access to the intervention. During ANC visits, pregnant women in addition to other health care services, are also provided with educational information about IPTp-SP and SP is offered to those who are due to receive SP. This could explain the higher IPTp-SP ideation noted among women who attended the recommended minimum number of ANC visits required for pregnant women. However, it should be noted two of the six items used to construct the IPTp-SP ideation are ANC related.

As would be expected, IPTp-SP ideation was significantly higher in women who reported to have been exposed to messages about malaria prevention within the past year compared to those who reported no exposure. The use of media messages to influence behavior change through its effect on ideation have been documented by prior research (Kincaid, 2000a; Babalola, Kusemiju, Calhoun, Corroon, \& Ajao, 2015).

Given the conservative nature of Northern Nigeria relative to the South, it was not surprising that women from Kebbi were significantly less likely to have higher IPTp-SP ideation compared to their counterpart from Akwa Ibom. Extant literature in the area of maternal and child health services have shown how ideational elements like social norms and attitudes, both of which are a function of sociocultural context influence use of preventive health care services (Antai, 2009; Babalola, \& Fatusi, 2009). Similarly, Babalola and colleagues have also reported lower contraceptive ideation among men in Kaduna, North West Nigeria compared to men in Ibadan, South West Nigeria (Babalola, Kusemiju, Calhoun, Corroon, \& Ajao, 2015).

This current study found a significant dose-response relationship between community level education and higher IPTp-SP ideation. Women residing in communities with the least proportion of secondary education were the least likely to have higher IPTp-SP ideation compared to their counterpart residing in communities where more women had at least a secondary education. Higher educational achievements have been shown to influence knowledge, perception and behavior. Indeed, several authors have documented the role of community level education in the adoption of contraceptive use and fertility preferences (Borenstein, Kendal, \& Feldman, 2006; Colleran, \& Mace, 2015).

\section{Conclusions and recommendations}

ANC attendance and IPTp-SP uptake were the major determinants of higher IPTp-SP ideation in this study. Given the abundance of literature that shows a positive link between ANC attendance and IPTp-SP uptake. It is possible that one of the pathways through which ANC attendance influences IPTp-SP uptake is by its effect on IPTp-SP ideation. In the light of this, interventions that are aimed at improving access to and quality of ANC services for pregnant women could significantly improve uptake of IPTp-SP through its effect on IPTp-SP ideation. Furthermore, the reciprocal relationship between behavior and ideation could be leveraged upon to improve IPTp-SP programming. In addition, the use of mass media to disseminate malaria prevention messages could be relevant in improving IPTp-SP ideation.

Given the effect of community level literacy rate and state of residence on IPTp-SP ideation, as found in this study, there is the need for audience segmentation in order to facilitate audience specific interventions aimed at improving IPTp-SP ideation. Finally, our findings suggests that the ideational model could be useful in the design and implementation of IPTp-SP interventions.

\section{Study limitations and strengths}

Like every other study, this study is not without some limitations. Firstly, the cross sectional nature of this study does not allow for causal inferences to be made. In spite of this, the large sample size and 
analysis technique employed in the study makes the findings relevant for program design and implementation.

The self-reported nature of the factors examined in this study, makes the findings subject to influences from recall and social desirability bias. However, proper training and supervision of data collectors, assuring the respondents of confidentiality and maintaining privacy during interviews were some of the measures used to minimize these effects.

\section{Acknowledgements}

This analysis was based on data collected with support of the US President's Malaria Initiative. The contents of this report are the sole responsibility of the authors and does not necessarily represent the views or positions of USAID, PMI, the U.S. Government, or The Johns Hopkins University.

\section{References}

[1].Antai, D. (2009). Inequitable childhood immunization uptake in Nigeria: a multilevel analysis of individual and contextual determinants. BMC Infectious Diseases, 9:181 doi: 10.1186/1471-2334-9-181.

[2].Babalola, S., \& Fatusi, A. (2009). Determinants of use of maternal health services in Nigeria - looking beyond individual and household factors. BMC Pregnancy and Childbirth, 9:43 doi: 10.1186/1471-2393-9-43.

[3].Babalola, S., John, S., Ajao, B., \& Speizer, I.S. (2015). Ideation and intention to use contraceptives in Kenya and Nigeria. Demographic Research, 33 (8) 211-238. DOI: 10.4054/DemRes.2015.33.8.

[4].Babalola, S., Ricotta, E., Awantang, G., Lewicky, N., Koenker, H., \& Toso, M. (2016). Correlates of IntraHousehold ITN Use in Liberia: A Multilevel Analysis of Household survey Data. PLoS ONE 11 (7): e0158331. doi:10.1371/journal.pone.0158331.

[5].Babalola, S., Kusemiju, B., Calhoun, L., Corroon, M., \& Ajao, B. (2015).Factors associated with contraceptive ideation among urban men in Nigeria. International Journal of Gynecology and Obstetrics 130; E42-E46.

[6].Babalola, S., Vondrasek, C., Brown, J., \& Traoré, R. (2001). The impact of a regional family planning service promotion initiative in Sub-Saharan Africa: evidence from Cameroon. Int Fam Plan Perspect, 27(4):186-216.

[7].Bajwa, S.K., Bajwa, S.J., Ghai, G.K., Singh, K., \& Singh, N. (2012). Knowledge, attitudes, beliefs, and perception of the North Indian population toward adoption of contraceptive practices. Asia Pac J Public Health, 24(6):1002-12.

[8].Borenstein, E., Kendal, J., \& Feldman, M. (2006). Cultural niche construction in a metapopulation. Theoretical population biology 70(1), 92-104.

[9].Colleran H, Mace R. 2015 Social network- and community-level influences on contraceptive use: evidence from rural Poland. Proc. R. Soc. B 282: 20150398. http://dx.doi.org/10.1098/rspb.2015.0398.

[10]. Desai, M., Ter Kuile, F.O., Nosten, F., McGready, R., Asamoa, K., Brabin, B., \& Newman, R.D. (2007). Epidemiology and burden of malaria in pregnancy. Lancet Infect Dis 7: 93-104.

[11]. Do, M., Babalola, S., Awantang, G., Toso, M., Lewicky, N., \& Tompsett, A. (2017). Associations between Malaria-Related Ideational Factors and Care-Seeking Behavior for Fever among Children under Five in Mali, Nigeria, and Madagascar. Forthcoming in Plos One.

[12]. Guyatt, H.L., \& Snow, R.W. (2001). The epidemiology and burden of Plasmodium falciparum-related anemia among pregnant women in sub-Saharan Africa. American Journal of Tropical Medicine and Hygiene, 64 (1-2 Suppl): 36-44.

[13]. Kaggwa E.B., Diop N., \& Storey, J.D. (2008). The role of individual and community normative factors: a multilevel analysis of contraceptive use among women in union in Mali. Int Fam Plan Perspect, 34(2):79-88.

[14]. Kasl, S.V., \& Cobb, S. (1966), Health Behavior, Illness Behavior and Sick Role Behavior. Archives of Environmental Health, 12(2) 246-66.

[15]. Kincaid, D.L. (2000a). Mass Media, Ideation, and Behavior A Longitudinal Analysis of Contraceptive Change in the Philippines. Communication Research 27(6): 723-763. doi: 10.1177/009365000027006003.

[16]. Kincaid, D.L. (2000b). Social networks, ideation, and contraceptive behavior in Bangladesh: a longitudinal analysis. Soc Sci Med, 50(2):215-31.

[17]. National Population Commission (NPC) [Nigeria] and ICF International. 2014. Nigeria Demographic and Health Survey 2013. Abuja, Nigeria, and Rockville, Maryland, USA: NPC and ICF International. 
DOI: 10.21522/TIJPH.2013.06.01.Art010

ISSN: $2520-3134$

[18]. National Malaria Elimination Programme (NMEP), National Population Commission (NPoPC), National Bureau of Statistics (NBS), \& ICF International. 2016). Nigeria Malaria Indicator Survey 2015: Key Indicators. Abuja, Nigeria, and Rockville, Maryland, USA: NMEP, NPoPC, and ICF International.

[19]. Newman, R.D., Robalo, M., Quakyi, I. (2004). Malaria during pregnancy: epidemiology, current prevention strategies, and future directions. Emerg Infect Dis. Available from http://dx.doi.org/10.3201/eid1011.040624_09. Accessed October 2016. DOI: 10.3201/eid1011.040624_09.

[20]. Nduka, F.O., Egbu, A., Okafor, C., Nwaugo, V.O. (2006). Prevalence of malaria parasites and anaemia in Pregnant and Non Pregnant women in Aba and Okigwe towns of Southeast Nigeria. Animal Research International 3(3): $508-512508$.

[21]. Nnaji G.A., Ikechebelu, J.I., Okafor, C.I. (2009). A comparison of the prevalence of malaria parasitaemia in pregnant and non-pregnant women. Niger J Med 18(1):47-51.

[22]. Ricotta, E.E., Boulay, M., Ainslie, R., Babalola, S., Fotheringham, M., Koenker, H., \& Lynch, M. (2015). The use of mediation analysis to assess the effects of a behavior change communication strategy on bed net ideation and household universal coverage in Tanzania. Malaria Journal, 14:15 DOI 10.1186/s12936-014-05310 .

[23]. Russell C.L, Sallau, A., Emukah, E., Graves, P.M., Noland, G.S., Ngondi, J.M.,..... Patterson E.A. (2015). Determinants of Bed Net Use in Southeast Nigeria following Mass Distribution of LLINs: Implications for Social Behavior Interventions. PLoS ONE 10(10): e0139447. doi:10.1371/journal.pone.0139447.

[24]. Ryan, S., Franzetta, K., \& Manlove, J. (2007). Knowledge, perceptions, and motivations for contraception: Influence on teens' contraceptive consistency. Youth Soc, 39(2):182-208.

[25]. Samandari G, Speizer I.S., \& O'Connell K. (2010). The role of social support and parity in contraceptive use in Cambodia. Int Perspect Sex Reprod Health, 36(3):122-31.

[26]. Sirima, S.B., Cotte, A.H., Konaté, A., Moran, A.C., Asamoa, K., Bougouma, E.C., Newman, R.D. (2006). Malaria prevention during pregnancy: Assessing the disease burden one year after implementing a program of intermittent preventive treatment in Koupéla District, Burkina Faso. Am J Trop Med Hyg, 75(2): 205-211.

[27]. Steketee, R.W., Nahlen, B.L., Parise, M.E., \& Menendez, C. (2001). The burden of malaria in pregnancy in malaria-endemic areas. American Journal of Tropical Medicine and Hygiene, 64(1-2 Suppl.): 28-35.

[28]. Steketee, R.W., Wirima, J.J., \& Campbell, C.C., (1996). Developing effective strategies for malaria prevention programmes for pregnant African women. American journal of tropical medicine and hygiene, 55 (1 Suppl): $95-100$.

[29]. Sutton, S. (2004). Determinants of Health-Related Behaviors: Theoretical and Methodological Issues. The SAGE Handbook of Health Psychology 94-126. Available at https://www.phpc.cam.ac.uk/pcu/wpcontent/files/2012/01/Ch-04.pdf Accessed May 162017.

[30]. Tay S.C.K., Agboli, E., Abruquah, H.H., Walana, W. (2013). Malaria and Anaemia in Pregnant and NonPregnant Women of Child-Bearing Age at the University Hospital, Kumasi, Ghana. Open Journal of Medical Microbiology, 3: 193-200.

[31]. Van de Kaa, D.J. (1996). Anchored Narratives: The Story and Findings of Half a Century of Research into the Determinants of Fertility. Population Studies: A Journal of Demography 50(3): 389-432. doi: 10.1080/0032472031000149546.

[32]. World Health Organization. (2004). A strategic framework for malaria prevention and control during pregnancy in the African region. Brazzaville, World Health Organization, Regional Office for Africa, 2004 (AFR/MAL/04/01). Available from http://whqlibdoc.who.int/afro/2004/AFR_MAL_04.01.pdf. Accessed June 22016.

[33]. World Health Organization. (2014). WHO policy brief for the implementation of intermittent preventive treatment of malaria in pregnancy using sulfadoxine-pyrimethamine (IPTp-SP) April 2013 (rev. January 2014. 